\title{
VÉGÉTATION FIXÉE ET PHOSPHORE EN PETIT COURS D'EAU. CONSÉQUENCES D'UNE RÉDUCTION DES APPORTS DE PHOSPHORE.
}

\author{
Marie-Christine PELTRE (1), L. LEGLIZE (1), J.L. SALLERON (2)
}

(1) Université de Metz, Laboratoire d'Ecologie, BP 4116, 57040 METZ Cedex.

(2) Agence de l'Eau Rhin-Meuse, BP 19, 57161 ROZERIEULLES.

\section{RÉSUMÉ}

Le cours d'eau du Vair, affluent de la Meuse, reçoit des effluents phosphorés dès l'amont, dans un contexte géologique complexe déterminant une forte minéralisation des eaux. D'autre part, son lit est fortement dégradé par un aménagement antérieur (curage, recalibrage).

Un développement important d'algues filamenteuses et de potamot pectiné à l'amont influence notablement la qualité de l'eau. De plus, on constate dans ce cours d'eau des blooms à Diatomées s'étendant jusqu'en Meuse aval.

Une réduction des apports de phosphore a été envisagée et mise en oeuvre. Un suivi de ces rejets et de leur impact sur la végétation a été mis en place en 1987 et 1988.

Les résultats mettent en évidence une diminution importante des flux de phosphore. Toutefois, on ne remarque pas de modification immédiate des descripteurs de la végétation. En revanche, l'étude du profil longitudinal de la rivière rend compte d'une amélioration sensible de la qualité générale du cours d'eau de l'amont vers l'aval.

Des actions permettant des réductions supplémentaires de ces apports et la restauration du lit sont engagées pour prolonger cette démarche qui restait encore insuffisante pour avoir un impact sur les proliférations végétales observées.

Mots-clés : Eutrophisation, cours d'eau, macrophytes, phosphore, maîtrise.

\section{AQUATIC VEGETATION AND PHOSPHORUS CONTENTS IN SMALL RIVER. CONSEQUENCES OF A REDUCTION IN PHOSPHORUS LEVELS}

\section{ABSTRACT}

The River Vair, a tributary of the Meuse, receives phosphorus effluents from upstream, in a complex geological process leading to the high mineralisation of river water. Also, the river bed has been previously degraded by a process of scrapping and remodelling.

A sizeable development of filamentous algae and Potamogeton pectinatus upstream, notably influences the water quality. Moreover, diatom blooms are observed in the river and propagate until flowing into the Meuse downstream.

A reduction in phosphorus levels was envisaged and carried out. A survey of wastewater and its impact on the vegetation was also carried out in 1987 and 1988.

The results show a considerable decrease in phosphorus loads. However, no immediate changes in the vegetation descriptors were found. A longitudinal profile study of the river shows a noticeable improvement of water quality from upstream to downstream. 
Further reductions of phosphorus levels and bed restoration are being undertaken to extend the study, as reductions were no sufficient enough to have an impact on observed vegetal proliferations.

Key-words : Eutrophication, river, macrophytes, phosphorus, control.

\section{INTRODUCTION}

La prolifération de certains végétaux aquatiques fixés en eau courante est un phénomène de plus en plus répandu, notamment dans les cours d'eau de moyenne importance. Ces développements excessifs sont souvent mis en relation avec un enrichissement général des eaux par les nutriments azotés et phosphorés (AGENCE DE L'EAU LOIRE-BRETAGNE, 1988), mais d'autres facteurs sont reconnus comme responsables ou aggravants (HASLAM, 1990).

La régulation de la biomasse phytoplanctonique en rivière peut être obtenue principalement par la maîtrise des rejets phosphorés, couplée à une prise en compte de paramètres tels que l'ensoleillement, la température de l'eau, la turbidité et le temps de séjour (GOSSE et al., 1986).

Le contrôle des proliférations à macroalgues, telle la Cladophore, peut aussi s'obtenir en gérant les rejets de nutriments, notamment le phosphore, mais ces expérimentations ont surtout été menées en lac (AUER et al., 1982 ; PAINTER et KAMAITIS, 1987).

La réaction des macrophytes à une réduction des apports du milieu en phosphore est, elle, très liée à la physiologie de la plante et à ses exigences. Le développement des macrophytes enracinés est fréquemment lié à la biodisponibilité du phosphore des sédiments (BLAKE, 1988).

Certains facteurs, tels la vitesse du courant, la profondeur, l'éclairement, sont impliqués à des degrés divers, dans le développement des macrophytes (DAWSON, 1988). L'ombrage des cours d'eau peut même jouer le rôle de facteur limitant principal (CANFIELD et HOYER, 1988). Cependant, selon les espèces, composition des sédiments et éclairement peuvent avoir un impact nuancé (CHAMBERS et KALFF, 1987).

II était intéressant d'aborder cette problématique à partir d'un cas concret comme le Vair, affluent de la Meuse amont, qui présente un ensemble de caractéristiques exemplaires :

- on constate depuis quelques années sur le bassin amont de ce cours d'eau, un développement presque exclusif et important d'algues filamenteuses et de potamot pectiné, ayant un impact certain sur la qualité de l'eau (cycles d'oxygène..)(GOSSE, 1989),

- on a remarqué par ailleurs en octobre 1986, que l'aval de ce cours d'eau était à l'origine de colorations brunes, dues à des développements planctoniques intenses (blooms dus à la Diatomée Stephanodiscus hantzschii)(PIERRE,1987), se reproduisant depuis plusieurs années dans toute la Meuse aval,

- cette rivière coule, entre le secteur de Vittel-Contrexéville et Neufchâteau, sur une succession de substrats géologiques variés, dont les caractéristiques conditionnent fortement la physico-chimie de l'eau (forte minéralisation en particulier)(S.R.A.E., 1975),

- des rejets importants, notamment phosphorés, liés à l'activité du secteur amont Vittel-Contrexéville, accentuent cet enrichissement naturel (LEGLIZE et SALLERON, 1988),

- certains aménagements antérieurs du lit (curages) ont aggravé cette situation, surtout sur la partie amont du cours (et recalibrages).

Au vu de cette situation, un programme d'action réduisant les apports de phosphore, fut proposé aux décideurs locaux par l'Agence de l'Eau Rhin-Meuse. Un protocole de suivi des rejets phosphorés et de la végétation aquatique a été mis en place sur ce système (LEGLIZE et PELTRE, 1988, 1990) : le bassin versant du Vair fut étudié et présenté avec ses caractéristiques abiotiques (géologie, hydrologie, physico-chimie de l'eau..), et biologiques, plus spécifiquement sa végétation aquatique. De plus, une expérimentation s'appuyant sur un bilan global du phosphore, a cherché à mettre en évidence les effets d'une réduction des apports phosphorés, engagée au niveau du bassin amont sur les différents compartiments étudiés. 


\section{PRÉSENTATION DU COURS D'EAU ET DE SON CONTEXTE}

\subsection{Présentation climatique, géologique et hydrologique}

Le Vair, affluent rive droite de la Meuse en amont de Neufchâteau, prend sa source en amont de la ville de Contrexéville. Sur un parcours d'une soixantaine de kilomètres, il reçoit ses principaux affluents : le Petit Vair, la Vraine et la Frezelle (Figure 1).

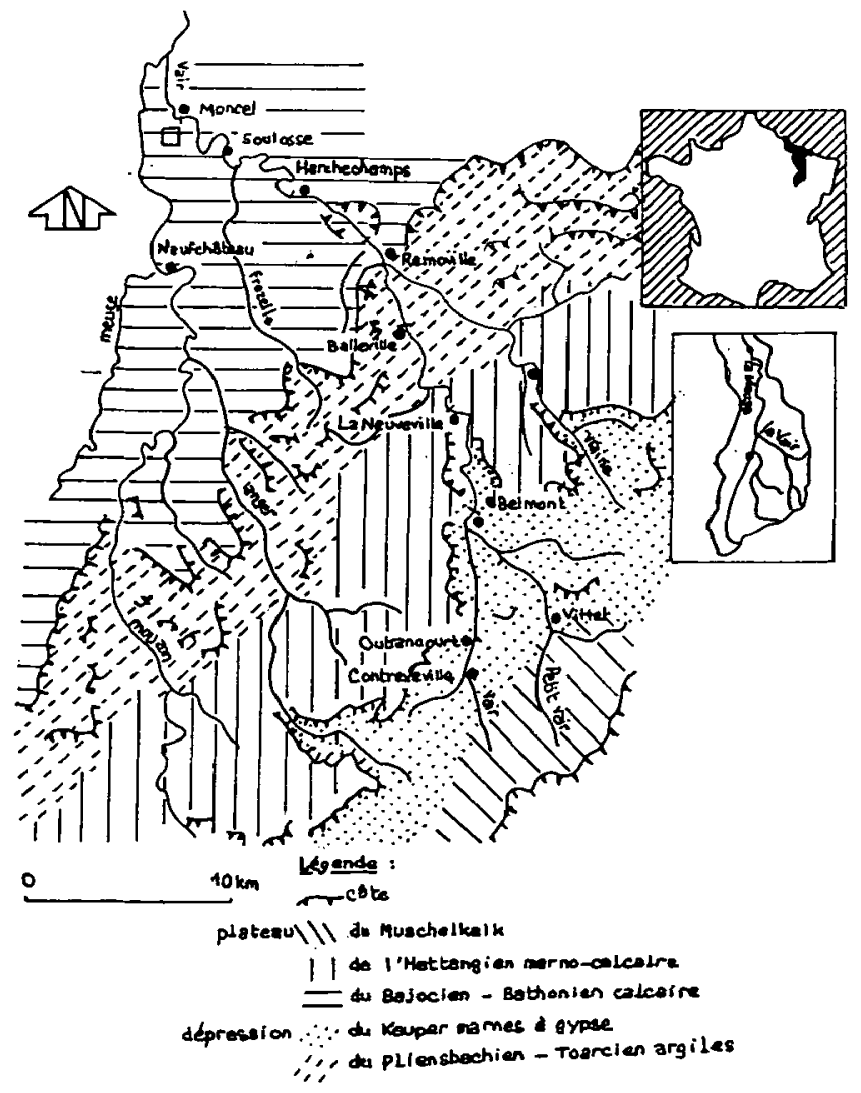

Figure 1 : Carte géomorphologique et localisation des stations du Vair. Figure 1 : Geomorphological map and location of the river Vair stations.

Le climat est de type continental, avec des pluies bien réparties sur toutes les saisons (pluviosité mensuelle autour de $70 \mathrm{~mm}$ ) et une température moyenne annuelle de $8-9^{\circ} \mathrm{C}$.

Le cours d'eau traverse, de l'amont vers l'aval, des régions à géologie différenciée : le Muchelkalk très en amont, les marnes du Keuper, l'Hettangien marno-calcaire, les argiles du Pliensbachien-Toarcien, et enfin à l'aval, les calcaires du Bajocien-Bathonien.

Cette succession de strates à caractère calcaire et argileux a façonné un relief de plateaux parfois karstifiés, de côtes et dépressions.

Diverses études (Agence de l'Eau Rhin-Meuse, 1989) font état d'une situation hydrologique spécifique fonction de la nature du substratum géologique drainé, avec dans les plateaux calcaires (amont de Contrexéville et aval du bassin versant), des échanges avec la nappe contenue dans le karst. Ces phénomènes favorisent des infiltrations et des apports qui peuvent modifier les caractéristiques physico-chimiques de l'eau.

\subsection{Données socio-économiques et d'aménagement}

Le bassin amont du Vair est marqué par une forte pression humaine. II se caractérise principalement (S.R.A.E., 1975), par : $30 \%$ du bassin,

- une occupation des sols de type agricole tournée vers l'élevage, où la forêt occupe 

Contrexéville,

- une démographie hétérogène où $47 \%$ de la population est concentrée à Vittel et

- des contraintes économiques, avec une activité thermale importante de l'amont du bassin versant, grâce aux sources hydrominérales froides de Vittel et Contrexéville. Les industries sont représentées par les deux Sociétés d'Eaux Minérales de Vittel et Contrexéville, et par d'autres entreprises liées aux activités agro-alimentaires du secteur (laiteries, conserveries, scieries...).

En complément, il faut signaler une forte dégradation des caractéristiques naturelles du lit, liées notamment au remembrement, surtout sur l'amont : suppression d'arbres, pentes abruptes, rectification et recalibrage du cours, l'affluent le Petit Vair étant tout particulièrement destructuré.

\subsection{Caractéristiques physico-chimiques}

Plusieurs campagnes d'étude (S.R.A.E., 1975 ; GOSSE, 1989), et les données du Réseau National de Bassin Rhin-Meuse, dressent une image des caractéristiques physicochimiques du Vair. Celles-ci sont largement influencées par les terrains géologiques qu'il traverse (Tableau I) : conductivité élevée (de l'ordre de $1000 \mu \mathrm{S} . \mathrm{cm}^{-1}$ sur le secteur amont), $\mathrm{pH}$ voisin de 8 , caractéristique d'eaux circulant en impluvium marno-calcaire, concentrations en sulfates très élevées, teneurs en phosphates rarement inférieures à $1000 \mu \mathrm{g} \mathrm{P} / \mathrm{l}$, voisinant les $3000 \mu \mathrm{g} \mathrm{P/l}$ dans le Petit Vair (en 1984).

En 1987, les rejets du Vair représentent $20 \%$ du total des rejets phosphorés en amont de Verdun (LEGLIZE et SALLERON, 1988).

Tableau I : Caractéristiques physico-chimiques du Vair : moyennes annuelles et valeurs extrêmes du R.N.B. 1991 (données Agence de l'Eau Rhin-Meuse).

Table I : Physico-chemical characteristics of the river Vair : annual means and extreme values of the R.N.B. 1991 (Water Agency Rhin-Meuse data).

\begin{tabular}{|c|c|c|c|c|c|c|c|}
\hline Stations & $\mathbf{p H}$ & $\begin{array}{c}\text { Conductivité } \\
\mu \mathrm{S} / \mathrm{cm}\end{array}$ & $\begin{array}{c}\text { Sulfates } \\
\mathrm{mg} / \mathrm{l}\end{array}$ & $\begin{array}{c}\mathrm{HCO} 3 \\
\mathrm{mg} / \mathrm{l}\end{array}$ & $\begin{array}{c}\text { Phosphates } \\
\mu \mathrm{g} \mathrm{P} / \mathrm{l}\end{array}$ & $\begin{array}{c}\text { Nitrates } \\
\mathrm{mg} / \mathrm{l}\end{array}$ & $\begin{array}{c}\text { Ammoniaque } \\
\mathrm{mg} / \mathrm{l}\end{array}$ \\
\hline Belmont & $\begin{array}{c}7,7 \\
(7,6 \cdot 7,9)\end{array}$ & $\begin{array}{c}1062 \\
(420-1300)\end{array}$ & $\begin{array}{c}354 \\
(240-400)\end{array}$ & $\begin{array}{c}384 \\
(251-451)\end{array}$ & $\begin{array}{c}900 \\
(290-1220)\end{array}$ & $\begin{array}{c}7,6 \\
(2,2-14,1)\end{array}$ & $\begin{array}{c}3,6 \\
(0,49-12,8)\end{array}$ \\
\hline Soulosse & $\begin{array}{c}8,2 \\
(7,8-8,4)\end{array}$ & $\begin{array}{c}780 \\
(195-1110)\end{array}$ & $\begin{array}{c}220 \\
(195-245)\end{array}$ & $\begin{array}{c}343 \\
(315-370)\end{array}$ & $\begin{array}{c}570 \\
(170-980)\end{array}$ & $\begin{array}{c}\mathbf{9 , 8} \\
(1,9-18,2)\end{array}$ & $\begin{array}{c}0,24 \\
(0,02-0,66)\end{array}$ \\
\hline
\end{tabular}

Une des spécificités de l'amont de ce cours d'eau concerne les variations très marquées des cycles journaliers de l'oxygène dissous et du pH en été (GOSSE, 1989) : des amplitudes d'environ $20 \mathrm{mg} \mathrm{O} / \mathrm{l}$ (avec parfois des concentrations en oxygène dissous proches de 0 le matin, et des taux de saturation pouvant dépasser $200 \%$ certains aprèsmidis), et des variations du $\mathrm{pH}$ supérieures à 1 unité $\mathrm{pH}$; en rivière naturellement alcaline, c'est l'indication d'une atteinte aigüe du milieu (BOUNI et LAURANS, 1991).

La contribution des algues épiphytes, nombreuses sur ces végétaux fixés (Diatomées), a d'ailleurs été démontrée dans leur participation à ces phénomènes (GOSSE, 1989).

\subsection{Etude de la végétation aquatique du Vair}

\subsubsection{Inventaire amont-aval des peuplements végétaux fixés}

Un profil floristique, effectué en septembre 1987, a permis de préciser la répartition spatiale des principaux groupements végétaux ainsi qu'une description phyto-sociologique de ceux-ci, affectant aux espèces le coefficient d'abondance-dominance de Braun-Blanquet (Tableau II).

Cette description a été complétée par une évaluation quantitative des biomasses fixées selon un échantillonnage aléatoire (10 quadrats de $33 \mathrm{~cm}^{3} /$ station). Quelques caractéristiques morphodynamiques du cours d'eau y sont associées. 
Tableau II : Profil floristique du Vair (Juillet 87).

Table II : Floristic profile of the river Vair (July 87).

\section{Coefficient d'abondance et de dominance de Braun-Blanquet}

Liste floristique

Pt Vair Vairam' Belm. Laneuv. Ball. Rain. Remov. Harch. Monc.

\begin{tabular}{|c|c|c|c|c|c|c|c|c|c|}
\hline Cladophora sp. & $3(2)$ & $5(5)$ & $2(4)$ & $4(4)$ & $1(2)$ & & $1(2)$ & + & $2(3)$ \\
\hline Phalaris arundinacea & 1 & 2 & 1 & 2 & 1(2) & & & + & + \\
\hline Scirpus lacustris v.fluitans & + & + & + & $1(2)$ & + & & + & $1(3)$ & + \\
\hline Potamogeton pectinatus & & & $3(4)$ & $1(3)$ & $2(3)$ & & $2(4)$ & $1(2)$ & 1 \\
\hline Nuphar lutea & & & & 1 & 1(3) & 1 & $1(2)$ & & + \\
\hline Scirpus lacustris & & & & 1 & + & + & & + & \\
\hline Fontinalis antipyretica & & & & 1 & + & & & + & + \\
\hline Vaucheria sp. & & & & + & & & + & & + \\
\hline Enteromorpha sp. & & & & + & & & + & + & 1 \\
\hline Sparganium erectum & & & & 1 & + & + & + & + & + \\
\hline Sagittaria sagittifolia & & & & & 1 & & + & + & + \\
\hline & & & & & + & 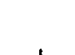 & + & + & + \\
\hline $\begin{array}{l}\text { Potamogeton crispus } \\
\text { Lycopus europeus }\end{array}$ & & & & & & $\begin{array}{l}+ \\
1\end{array}$ & & & + \\
\hline Alisma plantago-aquatica & & & & & & 1 & & + & \\
\hline Typha latifolia & & & & & & + & & & \\
\hline Sparganium simplex & & & & & & $1(3)$ & & & \\
\hline Myriophyllum spicatum & & & & & & & $2(2)$ & $2(2)$ & 2 \\
\hline Ranunculus fluitans & & & & & & & & & + \\
\hline Butomus umbellatus v. vallisn & & & & & & & & & + \\
\hline Iris pseudoacorus & & & & & & & & & + \\
\hline Biomasses g poids $\mathrm{sec} / \mathrm{m}^{2}$ & 78 & 672 & 196 & 173 & 21 & & 151 & 112 & 256 \\
\hline station $\mathbf{m}$ & 3,4 & 3,3 & 7,2 & 7,2 & 10,2 & & 10,6 & 9,3 & 27,5 \\
\hline Section station $\mathbf{m}^{2}$ & 1,09 & 0,73 & 3,32 & 2 & 2,9 & & 3,37 & 2,45 & 6,65 \\
\hline Vitesse moyenne $\mathrm{m} / \mathrm{s}$ & 0,16 & 0,32 & 0,145 & 0,18 & 0,21 & & 0,14 & 0,26 & 0,16 \\
\hline
\end{tabular}

L'inventaire des espèces récoltées montre un cortège des hydrophytes limité à une douzaine d'espèces assez communes :

- sur le Vair, en amont de la confluence Vair/Petit Vair, un développement presque exclusif de l'algue filamenteuse Cladophora sp. (et présence en automne de la bryophyte Amblystegium riparium) se traduit par une biomasse très forte à l'aval proche de Contrexéville ( $341 \mathrm{~g} . \mathrm{m}^{-2}$ exprimée en poids sec (PS) et un pourcentage de recouvrement de $75 \%$ ) ; et se réduit de moitié avant la confluence avec le Petit Vair.

- sur le Petit Vair, Cladophora sp. se développe également de façon monospécifique, mais présente une biomasse nettement plus faible $\left(77 \mathrm{~g} . \mathrm{m}^{-2} \mathrm{PS}\right.$ et $25-50 \%$ de recouvrement). Le substrat marneux ne favorise guère sa prolifération.

- après la confluence, à Belmont, Potamogeton pectinatus se développe fortement après la dominance printanière de Cladophora sp. et représente une forte proportion de la biomasse mesurée (196 g.m $\mathrm{m}^{-2} \mathrm{PS}$ ).

La richesse spécifique est donc très faible sur tout ce secteur amont (quelques individus de Scirpus lacustris et Phalaris arundinacea y sont rencontrés en complément).

Dès Laneuveville-sous-Châtenois, la richesse spécifique augmente, signe de l'amélioration de la qualité du milieu, avec une biomasse de 173 g.m $\mathrm{m}^{-2} \mathrm{PS}$, qui reste à un niveau élevé.

Ce processus se poursuit jusqu'à l'aval du cours d'eau, tout en se couplant avec des biomasses encore importantes. Potamogeton pectinatus et Cladophora sp. sont toujours présents, mais dans des proportions plus faibles. On voit progressivement apparaître Nuphar lutea, Myriophyllum spicatum, Ranunculus fluitans, d'autres algues filamenteuses (Enteromorpha sp., Vaucheria $s p$. ), ainsi que la bryophyte Fontinalis antipyretica. 


\subsubsection{Evolution phyto-planctonique}

La biomasse phytoplanctonique a fait elle aussi l'objet d'un suivi par une analyse des teneurs en pigments chlorophylliens. Les biomasses sont généralement inférieures à 5 mg.m ${ }^{-3}$ en chlorophylle "a" sur tout le cours du Vair, et comprises entre 5 et $10 \mathrm{mg}^{-3} \cdot \mathrm{m}^{-3}$ en chlorophylle totale. Le Petit Vair, lui, présente des valeurs supérieures à $10 \mathrm{mg} . \mathrm{m}^{-3}$, et des pourcentages de phéophytines souvent importants. Ces valeurs, sans être très élevées, ne sont pas anodines pour des amonts de cours d'eau (CROUZET et AMEZAL, 1982).

D'autre part, depuis plusieurs années, des épisodes importants de coloration brune des eaux de la Meuse apparaissent souvent en fin de période estivale. L'origine de cette apparition est observée dans le cours aval du Vair en octobre 1986. Le phénomène s'étend en quelques jours dans le cours de la Meuse jusqu'à Saint-Mihiel et au delà, avec des concentrations en chlorophylle "a" de plus de $100 \mathrm{mg} \cdot \mathrm{m}^{-3}$, et un pic atteignant $300 \mathrm{mg} \cdot \mathrm{m}^{-3}$. L'influence de ce phénomène se fait souvent sentir sur toute la Meuse aval jusqu'en Belgique (DESCY, comm.pers.).

PIERRE (1987) confirme l'ensemencement du cours aval du Vair et de la Meuse par une algue phytoplanctonique, la diatomée Stephanodiscus hantzschii. Celle-ci trouve dans ce milieu et à cette période de l'année, les conditions favorables à une prolifération importante (nutriments, température et produits de dégradation des macrophytes).

\section{NIVEAUX DES APPORTS DU BASSIN AMONT ET CONSÉQUENCES DE LEUR RÉDUCTION}

\subsection{Protocole de suivi}

Face à l'enrichissement excessif du cours d'eau, des solutions sont recherchées pour réduire les rejets. La première action dans ce domaine est réalisée en accord avec les gestionnaires, par la Société Générale des Eaux Minérales de Vittel, située en amont du Petit Vair, et dont les rejets représentent un pourcentage important des apports en phosphore de ce cours d'eau (LEGLIZE et SALLERON, 1988) : L'acide phosphorique utilisé dans les chaînes de lavage de l'usine d'embouteillage fut substitué par un mélange d'acide citrique et d'acide sulfurique tamponné et testé au cours de l'été 1987 (du 30 juillet au 25 septembre).

Cet essai s'étant avéré concluant, s'est prolongé par une expérimentation à long terme, du 20 mars 1988 à la mi-avril 1989.

Les apports en phosphore ont été mesurés avant et pendant les périodes de déphosphatation, dans l'eau, les sédiments et les végétaux.

L'influence sur le compartiment végétal a été appréciée par un suivi de la biomasse végétale sur les stations du cours amont : trois stations du Vair et celle du Petit Vair ont fait l'objet d'un suivi mensuel, de juillet à septembre 1987 et de mai à octobre 1988.

\subsection{Méthodologie}

Les méthodologies utilisées pour la prise en compte des différents paramètres sont développées ici :

\subsubsection{Le phosphore}

\section{- Dans l'eau}

Les ortho-phosphates et le phosphore total sont dosés selon la norme AFNOR (N.F.T 90023). En 1987, ils ont été analysés de façon ponctuelle, lors de quelques profils notamment.

En 1988, un suivi journalier du phosphore total a été effectué tout au long de l'année, à l'aval de la confluence Vair/ Petit Vair (Belmont). Couplé à un enregistrement limnigraphique, il a permis l'estimation de la charge en phosphore.

\section{- Dans les sédiments}

Les prélèvements des échantillons de sédiments s'effectuent, à plusieurs endroits différents de la station, à l'aide d'une drague à fond plat, selon le protocole normalisé. 
Le phosphore total et assimilable, l'azote Kjehldahl sont dosés sur sédiment séché (N.F.X 31-151).

\section{- Dans les végétaux}

L'analyse de la composition chimique des végétaux aquatiques s'effectue sur la plante entière après séchage à l'étuve (à $105^{\circ}$ pendant environ $24 \mathrm{H}$ ) et broyage sur la base d'un poids minimal de $5 \mathrm{~g}$. Les teneurs en $\mathrm{N}$ et $\mathrm{P}$ totaux sont exprimées en \% de poids sec.

\subsubsection{Etude de la biomasse végétale}

La technique d'échantillonnage adaptée à une population très peu variée, a utilisé un quadrat à armature métallique d'une superficie de $1 \mathrm{~m}^{2}$. L'intégralité des végétaux fixés à l'intérieur de la surface est récoltée (ne sont pas ramassés les végétaux fixés à l'extérieur et flottants à l'intérieur). Un seul quadrat est échantillonné par station.

La détermination de la biomasse s'effectue sur le terrain par une évaluation grossière du poids frais (PF), après essorage. Au laboratoire, les échantillons sont lavés soigneusement (pour éliminer une partie importante de la faune benthique qui s'abrite dans les macrophytes), et séchés à l'étuve à $105^{\circ} \mathrm{C}$ pendant environ $24 \mathrm{H}$, pour la mesure du poids sec (PS).

\subsection{Les apports phosphorés}

\subsubsection{Le phosphore dans l'eau}

* Distribution longitudinale des teneurs en phosphore dans l'eau en septembre 1987.

Cette distribution a été étudiée pendant la période de substitution lors d'une période hydrologique stable (Figure 2).

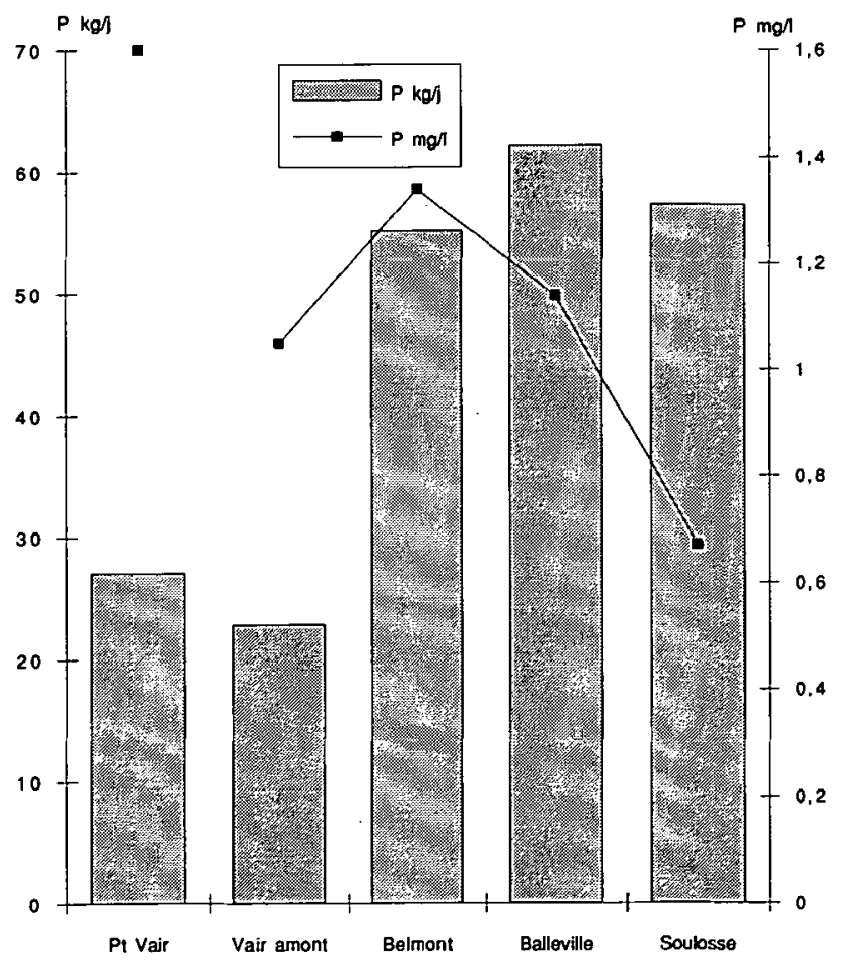

Figure 2 : Evolution longitudinale du phosphore total de l'eau (teneurs et charges) sur les stations du Vair. Campagne de septembre 1987.

Figure 2 : Longitudinal evolution of total phosphorus in water (concentrations and loads) on the river Vair stations. September sampling period 1987. 
Même pendant la période de substitution, les résultats mettent en évidence la prépondérance du Petit Vair au niveau des concentrations en phosphore (1.6 mg. $\left.\mathrm{I}^{-1}\right)$. Après l'arrêt des essais, une teneur importante de $2.9 \mathrm{mg} P / l$ de $P$ total est de nouveau observée dans celui-ci.

Les teneurs diminuent sensiblement vers l'aval, où l'on atteint $0.7 \mathrm{mg} . .^{-1} \mathrm{P}$ de phosphore total.

L'évolution des flux montre à Belmont une charge en $P(55 \mathrm{Kg} / \mathrm{j})$ équivalente à la somme des deux charges amont. Ils n'évoluent que peu jusqu'à la confluence avec la Meuse, sauf dans le secteur de Balleville où l'on observe une légère augmentation.

\section{* Suivi journalier 1988 à Belmont.}

La teneur moyenne annuelle en $P$ total est évaluée à $0.74 \mathrm{mg} . \mathrm{I}^{-1} \mathrm{P}$ (mini 0.15 , maxi

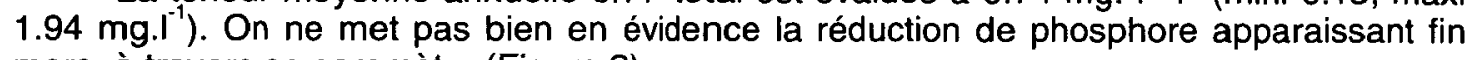
mars, à travers ce paramètre (Figure 3).

Par contre, l'évolution des flux de phosphore total met en évidence des charges élevées jusque fin mars (150-250 kg P/ jour), puis une très forte diminution dès le début du mois d'avril (50-66 kg P/j de moyenne jusqu'à la fin de l'année), reflet du début de l'abandon de l'acide phosphorique. On peut d'ailleurs mettre en parallèle ce niveau de flux avec celui atteint en septembre 1987 (55 kg P/j) lors de la phase d'essai.

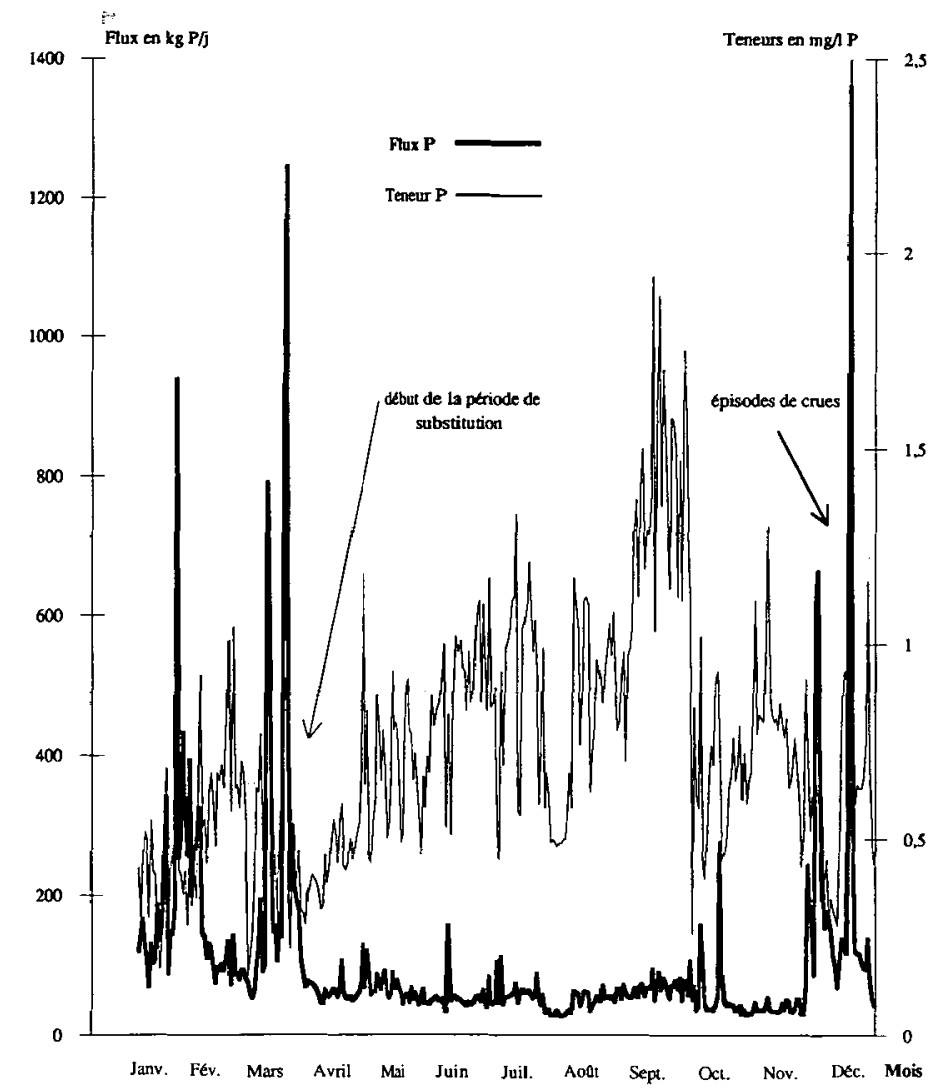

Figure 3 : Evolution journalière comparée des teneurs et des flux de phosphore total, à la station de Belmont en 1988.

Figure 3 : Daily evolution of total phosphorus contents and loads in water, at Belmont station, in 1988.

Durant cette période d'étude, il faut signaler lors d'épisodes de crues, l'augmentation brutale des charges en phosphore, par effet de lessivage des sols ou relargage des sédiments (DORIOZ et al., 1989). 
Comme on peut le remarquer, l'effort de réduction des charges en phosphore dès l'amont est une démarche indispensable. Cependant les teneurs résiduelles restent encore à un niveau élevé, mettant en évidence à la fois la nécessité de réduire davantage les rejets et celle de préciser les niveaux de phosphore des différents compartiments.

\subsubsection{Le phosphore dans les sédiments}

- Un bilan des teneurs en phosphore total des sédiments du cours amont du Vair, au cours de deux campagnes 1987-88, situe les valeurs relevées dans ce compartiment aux alentours de $0.27 \%$ du poids sec en P sur le Vair amont, et $0.15 \% \mathrm{P}$ sur le Petit Vair.

- Une étude de la teneur en phosphore total des sédiments lors du profil effectué en septembre 1987 (Figure 4), montre des concentrations variant de $0.1 \%$ à $0.28 \% P$ total de sédiment sec. Une décroissance amont-aval est visible, sauf dans le secteur de BallévilleHarchéchamp, où la nature géologique des marnes du Lias, riches en phosphates naturels, peut être mise en cause. On retrouve d'ailleurs une augmentation des charges en phosphore dans l'eau dans ce même secteur.

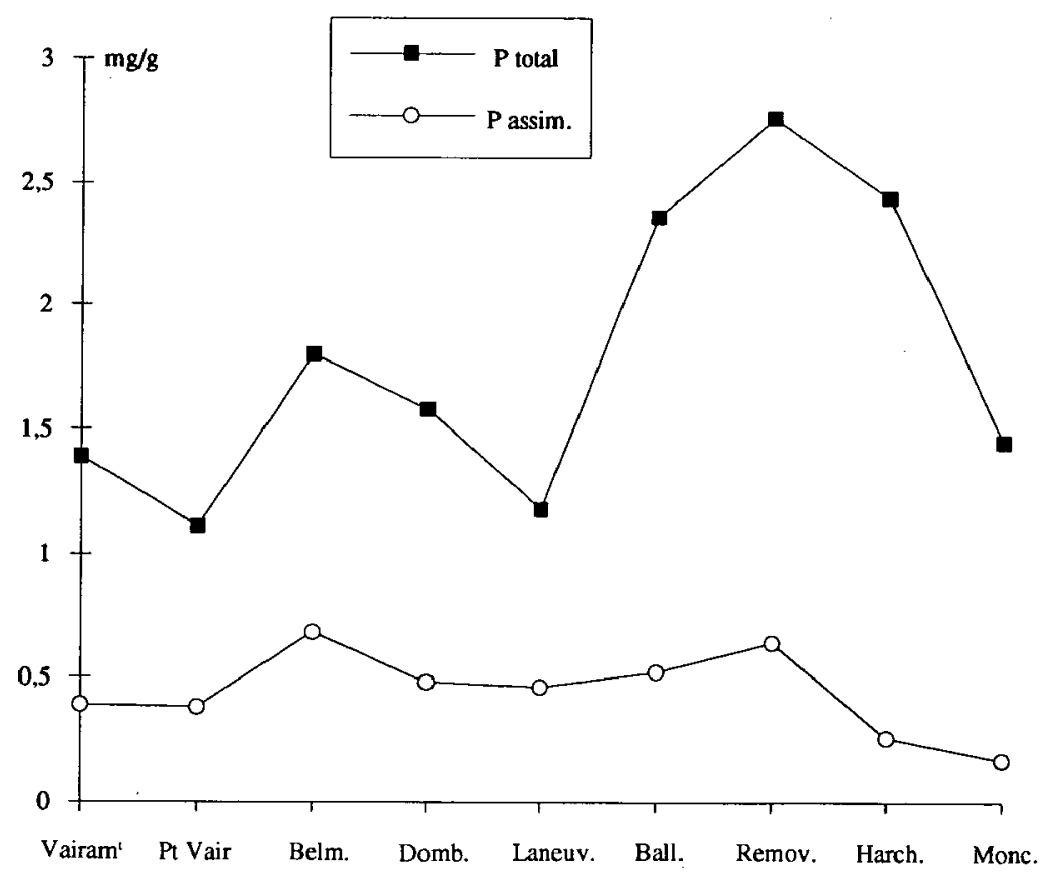

Figure 4 : Evolution longitudinale des teneurs en phosphore des sédiments sur le bassin du Vair (campagne de septembre 1987).

Figure 4 : Longitudinal evolution of total phosphorus contents in the Vair sediments (september sampling period 1987).

Les données de phosphore assimilable $\mathrm{P}_{2} \mathrm{O}_{5}$ dosées en 1987 correspondent à environ $1 / 3$ des teneurs en $P$ total, sauf sur le Vair amont et sur l'aval du cours d'eau où elles ne représentent pas plus du quart.

II n'y a pas de modification évidente des teneurs d'une année sur l'autre.

En référence à des dosages effectués sur un bassin versant expérimental (DORIOZ, 1989), ces données de phosphore dans les sédiments sont situées dans des gammes de teneurs élevées. 


\subsection{Influence de la réduction des rejets sur le compartiment végétal}

Une campagne d'expérimentation fut mise en place pour apprécier l'impact éventuel de la réduction des rejets phosphorés sur l'évolution des biomasses et sur les teneurs en nutriments des végétaux.

\subsubsection{Suivi temporel de la végétation fixée du secteur amont} recouvrement.

L'évaluation de la biomasse fixée a complété l'estimation visuelle du pourcentage de

Cette mesure, à l'aide d'un seul quadrat, pose souvent des difficultés dues à la représentativité de l'échantillonnage et à la variation temporelle des peuplements (HAURY et GOUESSE AIDARA, 1990). Cependant, l'erreur est moins importante lorsqu'on se situe comme ici dans des peuplements presque monospécifiques (GRASMUCK, 1989).

Les valeurs moyennes des biomasses (étés $87 / 88$ ) sont rapportées dans le Tableau III :

Tableau III : Evolution interannuelle des biomasses estivales du cours amont du Vair. Table III : Interannual evolution of summer biomass on the Vair uppercourse.

\begin{tabular}{lcccc}
\hline Stations & $\begin{array}{c}1984 \\
\text { (Juillet) }\end{array}$ & $\begin{array}{c}1987 / 88 \\
\text { (moy.) }\end{array}$ & $\begin{array}{c}1987 \\
\text { (moy. juil., } \\
\text { août, sep.) }\end{array}$ & $\begin{array}{c}1988 \\
\text { (moy. juil., } \\
\text { août, sep.) }\end{array}$ \\
\hline $\begin{array}{l}\text { Vair amont } \\
\text { Petit Vair }\end{array}$ & 110 & 200 & 300 & 102 \\
Belmont & 50 & 160 & 38 & 73 \\
Dombrot & 50 & 290 & $345(1$ val.) & 335 \\
\hline
\end{tabular}

N.B. : Biomasses exprimées en g. de poids $\mathrm{sec} / \mathrm{m}^{2}$.

Ces valeurs correspondent à un niveau globalement très élevé : WHITTON (1970) situe les biomasses maximales de cladophores aux alentours de $150-200 \mathrm{~g} . \mathrm{m}^{-2}$.PS. Les valeurs moyennes habituellement rencontrées se situent autour de $5-75 \mathrm{~g} . \mathrm{m}^{-2} \mathrm{PS}$ de moyenne annuelle (PITCAIRN et HAWKES, 1973).

Si on compare les biomasses moyennes de 1987 et 1988 , on note une diminution importante des valeurs moyennes sur le Vair amont et l'aval immédiat de la confluence en 1988, alors que le Petit Vair montre l'inverse (Tableau III). Une stabilisation apparaît à la station plus aval de Dombrot.

Une comparaison interannuelle avec les données de 1984 montre une diminution de moitié de la biomasse du Petit Vair, mais par contre une augmentation importante à l'aval de la confluence. Le protocole d'échantillonnage différent (en 1984, quadrat d'un $\mathrm{m}^{2}$ dans lequel tout ce qui est présent est récolté) peut sans doute influer sur la comparaison de ces résultats, mais pas au point de les contredire.

L'évolution saisonnière montre en 1987 une augmentation importante des biomasses de juillet à septembre sur les stations du Vair, mais une réduction sur le Petit Vair, situation non retrouvée en 1988. Une relation immédiate avec le test de déphosphatation ne peut donc être confortée.

\subsubsection{Teneurs en phosphore dans les végétaux}

Les teneurs rencontrées dans les végétaux du Vair $(0.6 \% \mathrm{P}$ pour Cladophora sp. et $0.45 \% \mathrm{P}$ pour Potamogeton pectinatus en moyenne) sont environ 10 fois supérieures aux moyennes généralement rencontrées (Tableau IV).

Une décroissance plus ou moins régulière, de l'amont vers l'aval, des teneurs en azote et phosphore total de Cladophora sp. et Potamogeton pectinatus est observée lors du 
profil de septembre 1987. Les teneurs en nutrimentś sont plus élevées à l'amont du Vair que sur le Petit Vair, et globalement plus élevées en 1988 qu'en 1987. II est à signaler que la méthodologie employée ne permet pas de séparer les teneurs dosées dans l'association épiphytes-macrophytes qui est importante sur ce secteur.

Tableau IV : Teneurs en nutriments relevées dans les végétaux du Vair. Table IV : Nutrient values in the river Vair plants.

\begin{tabular}{|c|c|c|c|c|c|}
\hline \multirow[t]{2}{*}{ Stations } & & \multicolumn{2}{|c|}{ Cladophora sp. } & \multicolumn{2}{|c|}{ Potamogeton pectinatus } \\
\hline & & phosphore & azote & phosphore & azote \\
\hline amont Vair & Sep-87 & 0,8 & 5,71 & 0,45 & 4,60 \\
\hline aval Vair & Sep-87 & 0,45 & 3,40 & 0,33 & 2,80 \\
\hline \multirow[t]{2}{*}{ Belmont } & moy. 1987 & 0,6 & & 0,45 & \\
\hline & moy. 1988 & 0,78 & & 0,59 & \\
\hline \multicolumn{6}{|l|}{ Bibliographie } \\
\hline WHITTON, 1970 & moy. rencontrées & 0,07 & & & \\
\hline AUER \& CANALE, 1982 & moy. rencontrées & 0,05 & & & \\
\hline GERLOFF\&FITZGERALD, 1976 & teneur mini nécess. & 0,06 & 1,1 & & \\
\hline HOWARD \& al.,1981 & & & & $0.02-0.13$ & \\
\hline \multirow[t]{2}{*}{ GERLOFF\&KROMBOLZ, 1966} & teneurs mini diff. macrophytes & 0,13 & 1,3 & & \\
\hline & & \multicolumn{4}{|c|}{$\mathrm{P}$ ou $\mathrm{N}$ en $\%$ poids sec } \\
\hline
\end{tabular}

\subsection{Bilan de l'expérimentation}

L'impact de la réduction actuelle des rejets de phosphore ne semble pas être ressenti à l'amont du cours d'eau au niveau du compartiment végétal, ni par l'estimation des biomasses, ni par le dosage du phosphore dans les végétaux, ni même au niveau du compartiment sédiments. Par contre, pour tous ces paramètres, une amélioration amontaval de la qualité générale est très nette.

\section{DISCUSSION - CONCLUSION}

\subsection{Point global sur la qualité de la rivière}

Au vu des résultats recueillis, le Vair confirme sa condition de cours d'eau à fortes potentialités d'enrichissement naturel et d'origine anthropique.

Cependant, malgré l'aspect très chargé du secteur amont, la situation s'améliore vers l'aval, avec toutefois un niveau trophique élevé. Différents paramètres en sont témoins :

- diminution progressive des teneurs en phosphore total dans l'eau, mais stabilisation des charges,

- diminution progressive des taux de phosphore total dans les sédiments, sauf dans la zone géologique des marnes du Lias,

- diminution progressive des teneurs en phosphore et azote total dans les végétaux,

- augmentation de la richesse spécifique des macrophytes le long du cours d'eau.

Cette évolution longitudinale a pu être confirmée par l'utilisation d'indices macrophytiques appliqués à la végétation des cours d'eau anglais : l'indice de NEWBOLD et HOLMES (1987) évolue peu de l'amont vers l'aval (112-114), image d'un niveau trophique élevé (le maximum est à 150). Celui d'HARDING (1987) évolue de 8 à 72 et visualise l'amélioration spécifique citée ci-dessus (gamme de 0 à >100).

Le cours du Vair prouve donc, malgré une situation amont bien dégradée, ses capacités de restauration. Ceci permet de préjuger favorablement de l'impact d'une réduction complémentaire des rejets qui pourrait être mise en place, dans les limites d'un contexte naturel qu'il convient de préciser. 


\subsection{Place du Vair dans la biotypologie végétale locale}

L'Anger et le Mouzon (LEGLIZE et GRASMUCK, 1992), sont des cours d'eau voisins du Vair, coulant sur alternance de substrats marnes-calcaires (l'Anger), ou calcairesmarnes/argiles (le Mouzon). Ils ont subi des recalibrages, présentent un assez bon éclairement, une forte minéralisation (conductivité souvent $>1000 \mu \mathrm{S} / \mathrm{cm}$ ), des teneurs en phosphore non négligeables $\left(200-600 \mu \mathrm{g} . \mathrm{I}^{-1} \mathrm{P}\right)$ et des pollutions ponctuelles marquées.

On se retrouve donc dans un contexte abiotique de cours d'eau voisins, autorisant les comparaisons.

Ces rivières présentent globalement de forts recouvrements de végétation aquatique. De nombreuses espèces présentes sont caractéristiques d'eaux minéralisées comme Myriophyllum spicatum, Nuphar lutea et Sparganium emersum, et même d'eaux très minéralisées comme Zannichellia palustris, Potamogeton crispus et Potamogeton pectinatus.

Le peuplement algal n'est pas dominé par Cladophora sp., qui est tout de même présente, mais par Vaucheria sp. qui est bien développée.

II est intéressant de remarquer que l'on n'observe pas sur ces cours d'eau de prolifération très importante de Cladophora $s p$., ni de Potamogeton pectinatus, comme sur l'amont du Vair. lls présentent toutefois de nombreuses espèces communes avec celles du cours aval du Vair.

Cette comparaison montre l'intérêt de la connaissance biotypologique. Elle permet d'apprécier les limites d'un contexte naturel et par là même celles d'une opération de restauration.

\subsection{Impact des actions engagées}

La réduction des apports de phosphore engagée par la Société des Eaux de Vittel en 1987 est une démarche importante pour l'amélioration de la qualité de ce cours d'eau. Elle est assez bien mise en évidence par le suivi des charges en phosphore dans l'eau, mais est parfois perturbée par l'effet du lessivage des sols ou du relargage du phosphore des sédiments en période de crues.

Malgré cela, les charges en phosphore restent encore élevées dans l'eau (55 Kg P/j) et dans les sédiments $(0.15 \% \mathrm{P})$.

Une diminution des biomasses végétales du secteur amont du cours d'eau n'a pu être encore observée au cours de la saison estivale 1988, pas plus d'ailleurs qu'une augmentation de la diversité spécifique qui pourrait témoigner d'une amélioration.

On remarque seulement une légère décroissance amont-aval des teneurs en nutriments dans les végétaux du secteur amont (principalement Cladophora sp.), aussi bien en 1987 qu'en 1988, qui peut être considérée comme un signe encourageant.

La comparaison interannuelle confirme le fait que la station du Vair amont, proche de l'agglomération de Contrexéville, montre les biomasses végétales et les teneurs en nutriments des végétaux les plus élevées, avec une diminution de ces derniers avant la confluence avec le Petit Vair. Celui-ci présente des valeurs de biomasses de Cladophora $s p$. inférieures à celles des autres stations qui ont même fortement diminué depuis la dernière investigation de 1984. Un lien avec une réduction des charges en phosphore peut être envisagée, étant donné la dépendance de cette algue vis-à-vis des teneurs en nutriments dans l'eau.

Cependant la relation linéaire liant les teneurs en phosphore du compartiment aqueux et celles rencontrées dans Cladophora $s p$. (et Potamogeton pectinatus), signalée par WONG et CLARK (1976), n'est pas mise en évidence sur le Vair. Au delà de valeurs moyennes de $P$, il n'y a pas de linéarité entre les teneurs tissulaires et les concentrations du milieu. Ceci rejoint la notion de "consommation de luxe", correspondant au plateau de la courbe où le taux de nutriments augmente dans la plante sans croissance de celle-ci (GERLOFF, 1969). 
On peut donc imaginer voir apparaître une réduction de son développement, parallèlement à celle des rejets. Une forte réduction de la biomasse de Cladophora $s p$. et de la teneur moyenne de ses tissus en $P$ est apparue lors d'une expérience intéressante de réduction des teneurs en phosphore du lac Ontario sur une période de 10 ans (PAINTER et KAMAITIS, 1987).

Le développement de Potamogeton pectinatus est, lui, fortement lié aux teneurs en phosphore dans les sédiments (BARKO et SMART, 1980). Et là encore, les teneurs en phosphore n'ont pas atteint un seuil minimal suffisant pour réguler la croissance de cette espèce, ce qui provoque une végétation encore très abondante et peu diversifiée jusqu'à Dombrot.

L'acquisition d'informations sur le déterminisme des espèces proliférantes semble donc fondamentale pour affiner la compréhension de leur développement et, à plus ou moins long terme, leur maîtrise.

L'effort reste donc encore à soutenir au niveau de la réduction des charges en phosphore aussi bien sur l'amont du Vair que sur le Petit Vair. En aval des actions précitées, l'assainissement du secteur de Vittel-Contrexéville a été engagé, avec des objectifs ambitieux de dépollution en matière de phosphore. Sont en préparation, notamment, la création d'une station d'épuration intercommunale Vittel-Contrexéville (comportant un étage de déphosphatation), et la réhabilitation des habitats aquatiques des zones amont.

L'objectif final est de retrouver une certaine diversité biologique qui entraînera une amélioration de l'auto-épuration et un retour de certaines espèces, notamment piscicoles.

D'autre part, l'amélioration de la qualité de l'amont ne pourra avoir qu'un impact positif sur la qualité du cours aval, et par voie de conséquence sur celle de la Meuse, réduisant par là les risques de voir apparaître les proliférations planctoniques dont il reste encore à démontrer les mécanismes déclencheurs.

De nouveaux suivis seront mis en place après la réalisation de ces travaux, permettant de préciser à partir de quel moment et à quelles teneurs en phosphore on pourra observer une augmentation de la richesse spécifique de la végétation macrophytique parallèllement à l'amélioration de la qualité physique de milieu prévue.

\section{REMERCIEMENTS}

Nous remercions vivement J.P. DECLOUX, N. GRASMUCK, P. RICHARD, qui ont participé à la réalisation de cette étude.

\section{BIBLIOGRAPHIE}

AGENCE DE L'EAU LOIRE-BRETAGNE, 1988. Azote et phosphore : Origine, effets, élimination. $174 \mathrm{p}$.

AGENCE DE L'EAU RHIN-MEUSE, 1989. Mesure des débits d'étiage des cours d'eau du Bassin du Vair. Campagne qualité et catalogue des débits d'étiage 1988 / GEREEA.

AUER M.T., R.P. CANALE, H.C. GRUNDLER, Y. MATSUOKA, 1982. Ecological studies and mathematical modelling of Cladophora in lake Huron. 2) Phosphorus uptake kinetics. J.Great Lakes Res., 8, 84-92.

BARKO J.W., SMART R.M., 1980. Mobilization of sediment phosphore by submersed freshwater macrophytes. Freshw. Biol., 10, 229-238.

BLAKE G., 1988. Rôle des végétaux aquatiques vis-à-vis du phosphore dans les écosystèmes et les systèmes d'épuration. AIDEC, Colloq.,"Le phosphore, ses dérivés et leur comportement dans le milieu naturel". Dijon, 22/24 nov.1988: 1-14.

BOUNI C., LAURANS Y., 1991. Détermination pour la collectivité nationale des coûts et dommages de toutes sortes entraînés par l'eutrophisation des eaux. Etude Interagences, 1 vol., 333 p.t annexes. 
CANFIELD D.E., J.R. and M.V. HOYER, 1988. Influence of nutrient enrichment and light avaibility on the abundance of aquatic macrophytes in Florida streams. Canad. J.Fish. and Aquat.Sci., 45 (8), 1467-72.

CHAMBERS P.A., KALFF J., 1987. Light and nutrients in the control of aquatic plant community structure. I. In situ experiments. J. of Ecology, 75, 611-619.

CROUZET Ph., AMEZAL A., 1982. Méthodologie d'analyse des causes et remèdes à l'eutrophisation des cours d'eau. Mise au point sur le bassin de l'Indre (France). S.H.F. XVII Journées de l'hydraulique.

DAWSON F.H., 1988. Water flow and the vegetation of running waters, 283-309, In Symoens J.J. : Vegetation of inland waters, 385 p. Kluwer Acad. Pub., Dordrecht.

DORIOZ J.M., PILLEBOUE E., FERHI A.,1989. Dynamique du phosphore dans les bassins versants : Importance des phénomènes de rétention dans les sédiments. Wat. Res., 23 (2), 147-158.

GERLOFF G.C., 1969. Evaluating nutrient supplies for the growth of aquatic plants in natural waters. In Eutrophication : Causes, consequences, correctives. Nat.Acad. Science, Washington D.C., 537-555.

GERLOFF G.C., FITZGERALD G.P., 1976. The nutrition of Great Lakes Cladophora. Unit. St. Environ. Prot. US EPA, Report. 600/ 3-76-044.

GERLOFF G.C., KROMBHOLZ P.H., 1966. Tissue analysis as a measure of nutrient availability for the growth of angiosperm aquatic plants. Limnol.Oceanogr., 11 (4), 529-537.

GOSSE Ph., 1989. Influence des végétaux fixés sur la qualité de l'eau d'une rivière : exemples du Vair et du Petit Vair en aval de Vittel. Rapport EDF Chatou DER/ HE31/89/1, $82 \mathrm{p}$.

GOSSE Ph., GRAS R.A., SALLERON J.L., LEGLIZE L., 1986. Approches successives dans le contrôle de l'eutrophisation d'une rivière : le Doubs, la Moselle et la Meuse. Soc. Hydrotechn. France, XIXe J. Hydraulique Paris, 9-11 sept. 1986, 8 p.

GRASMUCK N., 1989. Etude des relations entre végétaux aquatiques fixés et caractéristiques du milieu. Mem. DEA Sci. Agro., ENSAIA Nancy, 71 p.+ annexes.

HARDING J.P.C., 1987. The survey and assessment of macrophytes in watercourses, p.3453, in HMSO (Her Majesty's Stationery Office) : Methods for the use of aquatic macrophytes for assessing water quality 1985-86 : Methods for the examination of waters and associated materials. HMSO, London, $176 \mathrm{p}$.

HASLAM S.M., 1990. River pollution: An ecological perspective. Belhaven Press Publishers, London, $253 \mathrm{p}$.

HAURY J., GOUESSE AIDARA L., 1990. Etude méthodologique préliminaire de la biomasse des macrophytes en rivière. $14^{e}$ conf. COLUMA. J. Intern. Etudes sur le désherbage. Versailles, 247-255.

HOWARD-WILLIAMS C., ALLANSON B.R., 1981. Phosphorus cycling in a dense Potamogeton pectinatus. L.Bed.Oecol., 49, 56-66.

LEGLIZE L., PELTRE M.C., 1988. Végétaux aquatiques fixés et eutrophisation du bassin amont de la Meuse. Cas du Vair (été 1987). Rapport de contrat Agence de l'EAu Rhin-Meuse/ Univ.Metz, Laboratoire d'Ecologie. 69 p.+ annexes.

LEGLIZE L., PELTRE M.C., 1990. Etude des végétaux aquatiques sur le bassin amont du Vair (campagne estivale 1988). Rapport de contrat Agence de l'EAu Rhin-Meuse/ Univ.Metz, Laboratoire d'Ecologie. 25 p.+ annexes.

LEGLIZE L., GRASMUCK N., 1992. Etude des végétaux aquatiques des cours d'eau lorrains. Rapport de contrat Agence de l'Eau Rhin-Meuse/Univ.Metz, Laboratoire d'Ecologie. 42 p.+ annexes. 
LEGLIZE L., SALLERON J.L., 1988. L'eutrophisation en rivière. In L'impact de l'homme sur l'écosystème Meuse. JC MICHA \& S.PILETTE (eds). Press Univ.Namur, 33-38.

NEWBOLD C., HOLMES N.T.H., 1987. Nature conservation : water quality criteria and plants as water quality monitors. Water Pollut. Control, 86 (2), 345-364.

PAINTER D.S., KAMAITIS G., 1987. Reduction of Cladophora Biomass and Tissue Phosphorus in Lake Ontario, 1972-83. Canad. J. Fish. Aquat. Sc., 44 (12), 22122215.

PIERRE J.F., 1987. Flore algale et eutrophisation en Haute-Meuse. Bull. Acad. Soc. Lor. Sci., 26 (3), 91-100.

PITCAIRN C.E.R., HAWKES H.A., 1973. The role of phosphorus in the growth of Cladophora. Water Res., 7, 159-171.

SERVICE REGIONAL DE L'AMENAGEMENT DES EAUX EN LORRAINE, 1975. Monographie sommaire et qualité des eaux du Vair, $172 \mathrm{p}$. Ministère de l'Agriculture.

WHITTON B.A., 1970. Biology of Cladophora in freshwaters. Water Res., 4, 457-476.

WONG S.L., CLARK B., 1976. Field determination of the critical nutrient concentrations for Cladophora in streams. J.Fish Res. Board Can., 33 (1), 85-92. 